\title{
强碱催化分子内氮芳基化合成苯并咪唑反应机理及反应性研究
}

\author{
李强根*,a向仕凯 ${ }^{a}$ 毛双 ${ }^{a}$ 任 译*,b \\ $\left({ }^{a}\right.$ 四川师范大学化学与材料科学学院 成都 610068$)$ \\ $\left({ }^{b}\right.$ 四川大学化学学院 成都 610068)
}

\begin{abstract}
摘要 $\quad \mathrm{MP} 2 / 6-311+\mathrm{G}^{* *} / / \mathrm{B} 3 \mathrm{LYP} / 6-311+\mathrm{G}^{* *}$ *理论水平上, 对二甲基亚砜(DMSO)中强碱催化 $N$-(2-卤基苯基)- $N^{N}$-苯基乙 脒分子内氮芳基化合成苯并咪唑反应机理及反应性做了理论研究. 研究结果显示: 标题反应的反应机理并不是 Bolm 等提出的自由基机理或分步的 $\mathrm{S}_{\mathrm{N}} \mathrm{Ar}$ 机理，而是只有一个过渡态的协同 $\mathrm{S}_{\mathrm{N}} \mathrm{Ar}$ 机理. 几何结构宽松度分析和自然集居数 分析(NPA)都不能解释标题反应的反应性大小顺序问题. 多参数拟合揭示标题反应的反应能垒主要由最高占据轨道能

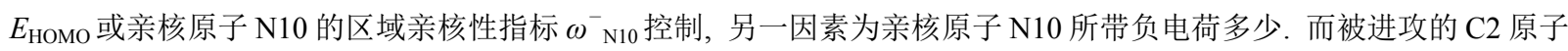
所带电荷以及几何结构宽松度 $L \%$ 对反应能垒几乎没有影响. 方法对比研究发现, MP2 $/ 6-311+\mathrm{G}^{* *} / / \mathrm{B} 3 L Y P / 6-311+\mathrm{G}^{* *}$ 方法所得结果与实验结果吻合较好, 能更好地描述标题反应的相对能量和反应性顺序.
\end{abstract}

关键词 分子内氮芳基化; $N$-(2-卤基苯基)- $N$ - -苯基乙脒; 反应机理; $\mathrm{S}_{\mathrm{N}} A r$ 反应; B3LYP; 亲核性指标

\section{Theoretical Investigations on the Intramolecular N-Arylation Mechanism and Reactivity for the Synthesis of Benzimidazoles by Base-Catalyzed}

\author{
Li, Qianggen ${ }^{* a} \quad$ Xiang, Shikai $^{a} \quad$ Mao, Shuang $^{a} \quad$ Ren, Yi* ${ }^{*}, b$ \\ ( ${ }^{a}$ College of Chemistry and Materials Science, Sichuan Normal University, Chengdu 610068) \\ ( ${ }^{b}$ College of Chemistry, Sichuan University, Chengdu 610064)
}

\begin{abstract}
Quantum chemical studies on the intramolecular $N$-arylation mechanism and reactivity of $N$-(2-halogen phenyl)$N$-phenyl ethyl amidines in dimethyl sulfoxide (DMSO) for the synthesis of benzimidazoles by base-catalyzed have been per-

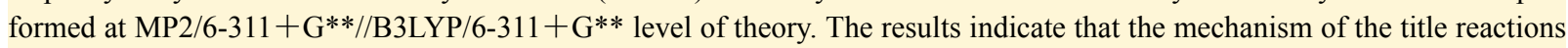
is not the radical mechanism or stepwise $\mathrm{S}_{\mathrm{N}} \mathrm{Ar}$ pathway, but the concerted $\mathrm{S}_{\mathrm{N}} \mathrm{Ar}$ pathway with a transition state, which is compared with the conclusion of Bolm et al. The reactivity of the title reactions can not be interpreted by the geometric looseness or natural population analysis. Multi parameter fitting reveals that the reactivity of the title reactions is controlled mostly by the regional nucleophilicity index $\omega^{-}$N10 of the nucleophile N10 atom or highest occupied molecular orbital energy $E_{\mathrm{HOMO}}$ of the reactant, the other factor is the charges of the nucleophilic atom N10, while the charges of the $\mathrm{C} 2$ atom and the geometric looseness $L \%$ have almost no effect on the reaction energy barrier. The relative energies and the reactivity of the title reactions

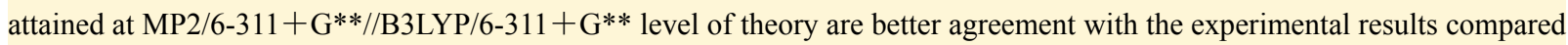
with the other methods.
\end{abstract}

Keywords intramolecular $N$-arylation; $N$-(2-halogen phenyl)- $N$-phenyl ethyl amidines; reaction mechanism; $\mathrm{S}_{\mathrm{N}} A r$ reaction; B3LYP; nucleophilicity index

苯并咪唑是一种拥有环化尿素骨架结构的重要杂 环化合物，其衍生物具有许多生物活性，药物市场上广 见其踪迹, 并且也是实验上的很多靶药 ${ }^{[1 \sim 5]}$. 因此, 苯
并咪唑的有效合成便是无数有机和药物化学家非常感 兴趣的领域之一 ${ }^{[6 ~ 51]}$. 传统的合成方法需要多步完成并 且收益不高 ${ }^{[6 \sim 8]}$. 近几十年发展了在高温下采用过渡金

* Corresponding authors. E-mail: liqgen@sina.com, renyi@scu.edu.cn

Received September 18, 2016; revised December 7, 2016; published online December 21, 2016.

Project supported by the National Natural Science Foundation of China (No. 21202109), the Research Fund of Department of Education, of Sichuan Province (No. 13ZB0160) and the Research Funds of Sichuan Normal University (No. 16ZP10).

国家自然科学基金(No.21202109)、四川省教育厅基金(No.13ZB0160)和四川师范大学基金(No. 16ZP10)资助项目. 
属催化合成苯并咪唑, 但是此工艺在制药工业应用中颇 有争议, 因为所得产物不纯净, 要去除杂质需要多步操 作, 并且需要合适的配体和造成环境污染 ${ }^{[19 ~ 32,49]}$. 最近 几个课题组发展了在强碱催化下, 通过分子内氮芳基化 来合成苯并咪唑和吲哚的新方法. 此方法的优点是不需 要过渡金属催化, 且反应条件温和, 产率高, 不需要多 步进行, 有的甚至一锅合成 ${ }^{[45 ~ 51]}$. 然而在反应机理上一 直存在争议. 由于反应在强碱中进行, 因此分子内氮芳 基化 $\mathrm{C}-\mathrm{N}$ 键耦合的初始物为 $N$-甲苯磺酰腙阴离子[49] 或 $N$-苯基取代脒阴离子 ${ }^{[50,51]}$, 目前其反应机理主要有四 种假设(图 1): (1)电环化机理 ${ }^{[49]},(2)$ 苯炔机理 ${ }^{[50]}$, (3)自由 基机理 ${ }^{[49,51]}$, (4)芳环上亲核取代机理 $\left(\mathrm{S}_{\mathrm{N}} \mathrm{Ar}\right)^{[51]}$. Bolm 等 ${ }^{[49]}$ 在前期通过分子内氮芳基化合成吲哚的工作中，提 出了电环化机理, 然而他们在实验中并没有观察到具有 四面体碳化合物阴离子存在, 因此排除了电环化机理, 但是他们通过电子自旋共振(ESR)光谱法观察到自由基 中间体存在, 因此认为该反应为双自由基机理. 2013 年 Xiang 等 ${ }^{[50]}$ 在通过分子内氮芳基化合成苯并咪唑的工作 中, 使用自由基捕获剂(TEMPO)后仍然发现有产物生 成, 因此认为不可能是自由基机理, 同时他们提出了苯 炔机理. 接着 Bolm 等 $^{[51]}$ 在通过分子内氮芳基化合成苯 并咪唑的工作中发现, 如果把反应物中的卤素取代位置 从邻位换到间位, 结果没有发现产物生成, 而把邻位与 间位都换成卤素取代基反而有产物生成, 并且反应活性 顺序为 $\mathrm{F}>\mathrm{I}>\mathrm{Br}>\mathrm{Cl}$, 实验结果与 $\mathrm{C}-\mathrm{X}$ 键极性大小顺 序 $\mathrm{X}=\mathrm{F}>\mathrm{Cl}>\mathrm{Br}>\mathrm{I}$ 矛盾, 因此认为不可能是苯炔机 理; 另外, 由于实验中反应活性大小顺序为 $\mathrm{F}>\mathrm{I}>\mathrm{Br}>$ $\mathrm{Cl}$, 与经典的活化芳环 $\mathrm{S}_{\mathrm{N}} \mathrm{Ar}$ 反应活性顺序 $\mathrm{F}>\mathrm{Cl}>$ $\mathrm{Br}>\mathrm{I}^{[52]}$ 不一致, 最后 Bolm 等 ${ }^{[51]}$ 得出, 对于离去基团是 $\mathrm{F} 、 \mathrm{Cl}$ 和 $\mathrm{Br}$ 的反应可能为 $\mathrm{S}_{\mathrm{N}} \mathrm{Ar}$ 亲核取代机理, 而离去 基团是 I 的反应可能为自由基机理.

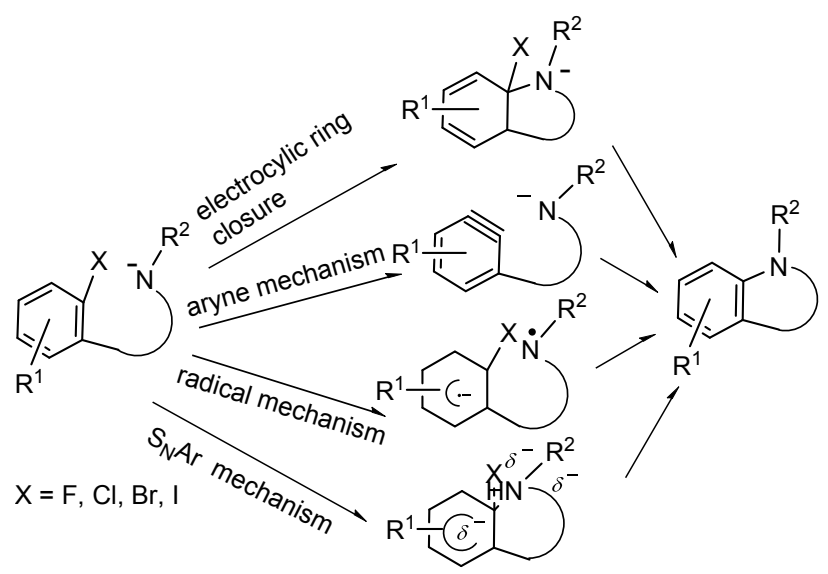

图 1 强碱催化分子内氮芳基化 $\mathrm{C}-\mathrm{N}$ 键耦合可能反应机理 Figure 1 Possible reaction mechanisms of intramolecular $\mathrm{N}$-arylation for the $\mathrm{C}-\mathrm{N}$ bond coupling reaction by base-catalyzed
对于芳环上的 $\mathrm{S}_{\mathrm{N}} \mathrm{Ar}$ 反应，通常指的是底物芳环临 位或对位上存在吸电子基团，即芳环是活化的情况，其 反应机理通常是加成-消去两步机理, 若离去基团是卤 素, 其反应活性顺序通常为 $\mathrm{F}>\mathrm{Cl}>\mathrm{Br}>\mathrm{I}$, 即加成是决 速步骤 ${ }^{[53]}$. 然而这一顺序会受亲核试剂和溶剂的影响, 例如对于一些软的, 高度可极化的亲核试剂 $\mathrm{SCN}^{-}$, $\mathrm{C}_{6} \mathrm{H}_{5} \mathrm{NHCH}_{3}$ 或 $\mathrm{NO}_{2}{ }^{-}$等, 反应活性顺序可能为 $\mathrm{I}>\mathrm{Br}>$ $\mathrm{Cl}>\mathrm{F}^{[53]}$. 除了加成 - 消去两步机理外, 少数芳环上的 $\mathrm{S}_{\mathrm{N}} \mathrm{Ar}$ 反应还存在只经过一个过渡态的协同机理, 如苯 酚与三嗪的反应 ${ }^{[54]}$, 以及甲醇阴离子与氯苯 ${ }^{[55]}$, 氟离子 与硝基苯 ${ }^{[56]}$, 对氧基氯苯或对氰基硝基苯与氟离子的 反应 ${ }^{[57]}$ 等，其反应机理都是协同的 $\mathrm{S}_{\mathrm{N}} \mathrm{Ar}$ 过程.

为了验证 Bolm 等 ${ }^{[51]}$ 提出的反应机理的合理性，同 时解释该反应的反应性大小顺序问题 $(\mathrm{F}>\mathrm{I}>\mathrm{Br}>\mathrm{Cl})$, 并揭示影响反应性大小的内在因素，本文以 $N$-(2-卤基 苯基)- $N$ '-苯基乙脒阴离子作为反应物 $\mathbf{R e}$ 出发, 设计了 四条可能的反应通道，对二甲基亚砜(DMSO)中强碱催 化分子内氮芳基化合成苯并咪唑反应机理做了理论探 索(图 2): Path 1 为单自由基机理; Path 2 为分子内电子转 移的双自由基机理, 并且考察了单重态和三重态双自由 基的可能性; Path 3 为先加成再消去的分步 $\mathrm{S}_{\mathrm{N}} \mathrm{Ar}$ 机理; Path 4 为协同的 $\mathrm{S}_{\mathrm{N}} \mathrm{Ar}$ 机理. 希望我们的研究结果能为下 一步的实验或理论研究提供有用的信息.

\section{2 计算方法}

采用密度泛函方法 $\mathrm{B} 3 \mathrm{LYP} \mathrm{P}^{[58 \sim 60]}$ 对所有物质几何结 构在实验实际进行溶剂二甲基亚砜(DMSO, $\varepsilon=46.7$ )中 进行优化. 选取此方法的原因是, B3LYP 方法是被广泛 认同并最为流行的密度泛函方法, 并且该方法在先前也 被多个课题组用来研究芳环上亲核取代反应 ${ }^{[61 ~ 68]}$. 为 了得到值得信赖的计算结果，我们先选择 $6-31+\mathrm{G}^{*}$ 基 进行优化, 接着再选择 $6-11+\mathrm{G}^{*}$ *进行优化, 目的是考 察基组的加大对几何结构与反应能垒的影响. 所有优化 的几何结构都用计算得到的频率来区别, 没有虚频的为 全程或局域稳定点, 有且只有一个虚频的为反应的过渡 态. 分别在 B3LYP/6-31+G* 和 B3LYP/6-311+G**理论 水平上计算获得频率，并且没有加任何的校正因子. 对 于含溴和碘的物质使用 Wadt 和 $\mathrm{Hay}^{[69]}$ 的有效核电势 (ECP)计算, 而对于其他物质, 采用全电子计算. 为了评 价 B3LYP 方法能量的精度, 我们还在 MP2/6-311+ $\mathrm{G}^{* * / / B} 3 \mathrm{LYP} / 6-311+\mathrm{G}^{* *}$ 和 MP3/6-311+ $\mathrm{G}^{* * / / B 3 L Y P} /$ $6-311+\mathrm{G}^{* *}$ 理论水平上, 采用连续介质模型 $(\mathrm{PCM})^{[70]}$ 进 行了液相单点能计算. 各物质的原子电荷以及键极参 数是基于自然键轨道 $(\mathrm{NBO})^{[71]}$ 的波函数计算，在 $\mathrm{MP} 2 / 6-311+\mathrm{G}^{* *} / / \mathrm{B} 3 \mathrm{LYP} / 6-311+\mathrm{G}^{* *}$ 理论水平上计算 


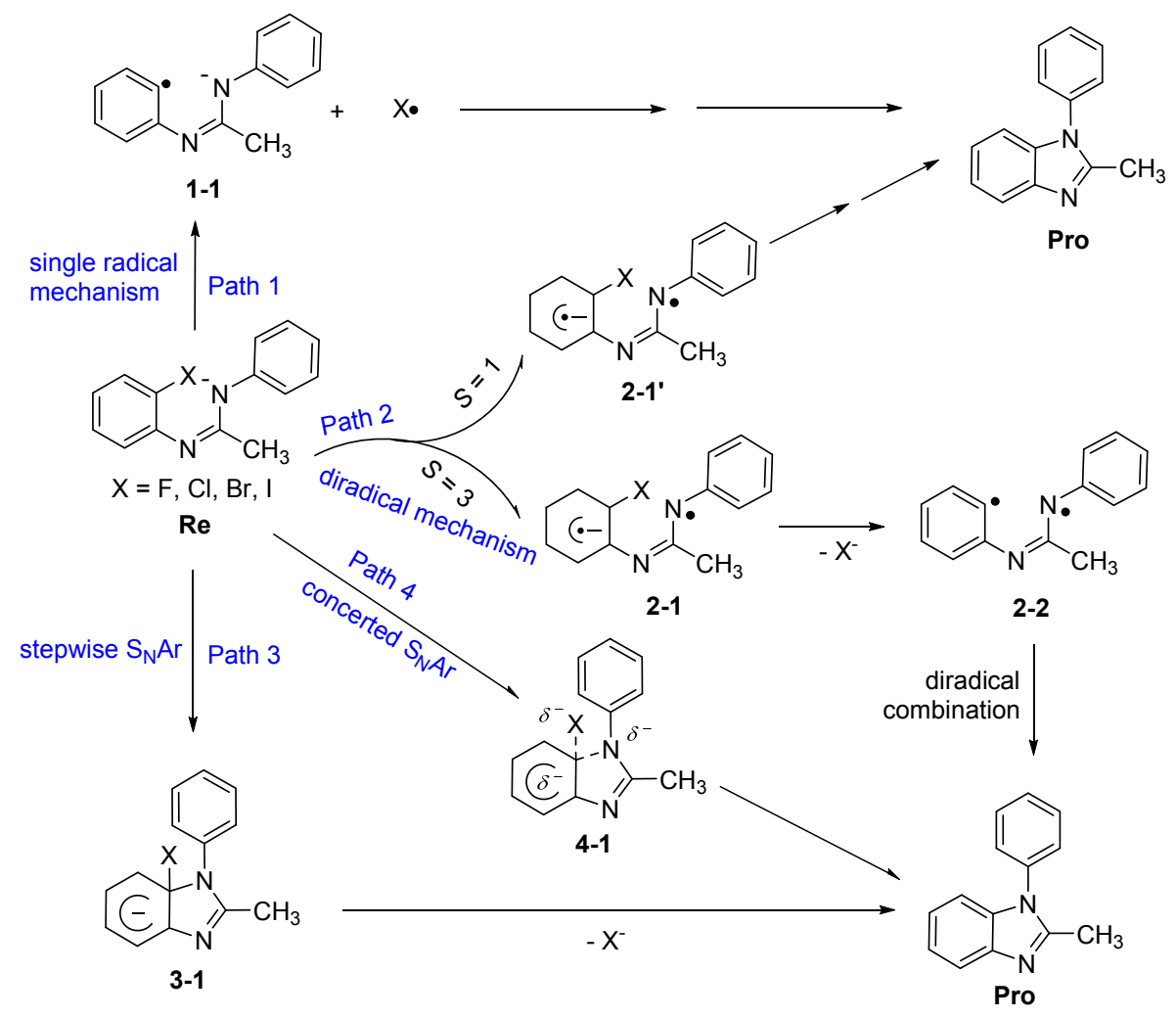

图 2 本文研究的分子内氮芳基化合成苯并咪唑可能反应通道

Figure 2 Possible reaction pathways in this study of intramolecular $N$-arylations of $N$-(2-halogen phenyl)- $N$-phenyl ethyl amidines for the synthesis of benzimidazoles

得到. 此外, 为了评价 B3LYP 方法的可靠性, 我们还使 用密度泛函方法 M06- $2 X^{[72]}$ 对所有物质几何结构在溶剂 DMSO 中进行优化. 同样在 MP2/6-311+ $\mathrm{G}^{* * / / M 06-2 X / ~}$ 6-311 $+\mathrm{G}^{* *}$ 理论水平上进行了液相 PCM 单点能计算. 选取该方法的原因是该方法在先前也曾被用来研究芳 环上亲核取代反应 ${ }^{[73 \sim 74]}$

本工作中, 所有的计算都是采用高斯 09 软件包 ${ }^{[75]}$ 完成, 所有原子核间距离都用纳米 $(\mathrm{nm})$ 而所有的键角都 用度 $\left(^{\circ}\right)$. 采用 $101 \mathrm{kPa}$ 下 $0 \mathrm{~K}$ 电子能变 $\left(\Delta E, \mathrm{~kJ}^{\circ} \cdot \mathrm{mol}^{-1}\right)$ 和 $298.15 \mathrm{~K}$ 的焓变 $\left(\Delta H, \mathrm{~kJ} \cdot \mathrm{mol}^{-1}\right)$ 以及吉布斯自由能变 $\left(\Delta G, \mathrm{~kJ} \cdot \mathrm{mol}^{-1}\right)$ 来表示相对能量.

\section{3 结果与讨论}

\section{1 自由基机理(Path 1 和 Path 2)}

对于自由基机理, 我们同时考察了单自由基和双自 由基机理两种情况(图 2). 在单自由基路径中(Path 1), 首先反应物 $\operatorname{Re}$ 中 $\mathrm{C}-\mathrm{X}$ 键发生均裂形成 1-1 和 $\mathrm{X}$ ·两个 单自由基, 然后 1-1 再关环形成产物. $\mathrm{C}-\mathrm{X}$ 键均裂能垒 总结在表 1 中, 从该表数据可以看出, 在 M06-2X/6$311+\mathrm{G}^{* *}$ 理论水平上, 从 $\mathrm{F}$ 到 $\mathrm{I}$ 能垒分别为 $466.6(\mathrm{X}=$ F), $349.9(\mathrm{X}=\mathrm{Cl}), 308.3(\mathrm{X}=\mathrm{Br})$ 和 $263.8 \mathrm{~kJ} \cdot \mathrm{mol}^{-1}(\mathrm{X}=$
I); 而在 MP2/6-311 $+\mathrm{G}^{* * / / M 06-2 X / 6-311+~} \mathrm{G}^{* *}$ 理论水 平上却高达 $557.8(\mathrm{X}=\mathrm{F}), 454.9(\mathrm{X}=\mathrm{Cl}), 394.8(\mathrm{X}=\mathrm{Br})$ 和 $351.5 \mathrm{~kJ} \cdot \mathrm{mol}^{-1}(\mathrm{X}=\mathrm{I})$, 因此反应物 $\mathbf{R e}$ 中 $\mathrm{C}-\mathrm{X}$ 键发 生均裂形成 1-1 和 $\mathbf{X}$ ·两个单自由基几乎不可能, 即标题 反应单自由基机理不可能存在. 对于双自由基机理, 根 据双自由基反应路径(Path 2), 反应物 $\operatorname{Re}$ 首先必须通过 电子转移生成双自由基阴离子, 同时讨论了单重态的双 自由基(2-1')和三重态的双自由基(2-1)的情况, 对于单 重态的双自由基(2-1'), 通过对反应物 Re加关键词 guess $=$ mix 进行优化, 并且对波函数的稳定性做了测试, 结 果得到的结构和能量与反应物 $\mathbf{R e}$ 一样, 且 $\left\langle\mathrm{S}^{2}\right\rangle=0$, 说明电子总自旋为 0 , 为闭壳层单重态, 而非双自由基, 即不存在双自由基单线态. 然而我们可以优化得到稳定 的三重态双自由基， $\left\langle\mathrm{S}^{2}>\right.$ 值分别为 $2.027(\mathrm{X}=\mathrm{F})$, $2.028(\mathrm{X}=\mathrm{Cl}), 2.0285(\mathrm{X}=\mathrm{Br})$ 和 $2.047(\mathrm{X}=\mathrm{I})$. 另外, 在 $\mathrm{MP} 2 / 6-311+\mathrm{G}^{* *} / / \mathrm{B} 3 \mathrm{LYP} / 6-311+\mathrm{G}^{* *}$ 理论水平上计算 得到反应物 $N$-(2-氟基苯基)- $N^{\prime}$-苯基乙脒阴离子及其相 应的三重态双自由基的 HOMO 轨道(图 3). 从图 3 可以 看出，单重态反应物 $N$-(2-氟基苯基)- $N$ '-苯基乙脒阴离 子中亲核原子 $\mathrm{N}$ 的 $\mathrm{p}$ 轨道与亲核基团的苯环的 $\pi$ 轨道存 在 $\mathrm{p}-\boldsymbol{\pi}$ 相互作用，而在三重态的双自由基阴离子中却不 
存在这种作用, 因此后者能量应该比前者要高. 表 1 总 结了标题反应所有反应物 $\operatorname{Re}$ (单重态)与双自由基阴离 子 2-1 (三重态) 的相对能量, 从该表数据可以看出, 在 M06-2X/6-311 $+\mathrm{G}^{* *}$ 理论水平上, 三重态的双自由基阴 离子比相应的反应物能量分别高出 $275.5(\mathrm{X}=\mathrm{F}), 260.6$ $(\mathrm{X}=\mathrm{Cl}), 260.4(\mathrm{X}=\mathrm{Br})$ 和 $253.3 \mathrm{~kJ} \cdot \mathrm{mol}^{-1}(\mathrm{X}=\mathrm{I})$; 而在

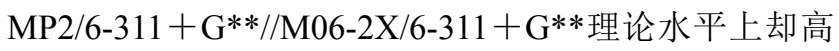
达 $463.3(\mathrm{X}=\mathrm{F}), 443.1(\mathrm{X}=\mathrm{Cl}), 435.5(\mathrm{X}=\mathrm{Br})$ 和 431.8 $\mathrm{kJ} \cdot \mathrm{mol}^{-1}(\mathrm{X}=\mathrm{I})$, 因此要从反应物 $\mathbf{R e}$ 生成相应的三重态 双自由基阴离子也是几乎不可能的. 从以上分析可以得 出, 标题反应的自由基机理几乎不可能存在.

\section{2 芳环上亲核取代机理 $\left(S_{N} A r\right.$, Path 3 和 Path 4)}

对于芳环上的亲核取代路径, 在几何结构优化过程 中, 没有得到 $\sigma$ 络合物而只得到协同的过渡态结构, 因 此标题反应不是通过先加成后消去的分步亲核取代过 程(Path 3), 而是一个协同的亲核取代机理(Path 4). 不 同水平上计算所得标题反应协同 $\mathrm{S}_{\mathrm{N}} \mathrm{Ar}$ 路径相对能量总 结在表 2 中, 而 B3LYP/6-311+G**理论水平上和 DMSO 溶剂中优化所得标题反应协同 $\mathrm{S}_{\mathrm{N}} \mathrm{Ar}$ 路径所有驻 点几何结构总结在图 4 中. 从表 2 能量数据可以看出, $\mathrm{MP} 2 / 6-311+\mathrm{G}^{* * / / B} 3 \mathrm{LYP} / 6-311+\mathrm{G}^{* *}$ 方法水平上计算 所得反应吉布斯自由能垒 $\left(\Delta G^{\ddagger}\right)$ 分别为 $90.5(\mathrm{X}=\mathrm{F})$,
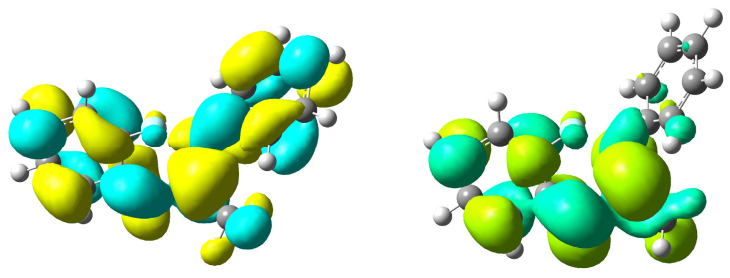

图 $3 \mathrm{MP} 2 / 6-311+\mathrm{G}^{* *} / / \mathrm{B} 3 \mathrm{LYP} / 6-311+\mathrm{G}^{* *}$ 理论水平上计算 得到反应物 $N$-(2-氟基苯基)- $N$ '-苯基乙脒阴离子(左)及其相应 的三重态双自由基阴离子(右)HOMO 轨道图

Figure 3 HOMO orbitals of the reactant $N$-(2-fluorine phenyl)- $N$ '-phenyl ethyl amidine anion (left) and its double radical anion (triplet state) (right) attained at MP2/6-311+ $\mathrm{G}^{* * / / \mathrm{B} 3 \mathrm{LYP} /}$ $6-311+\mathrm{G}^{* *}$ level

$109.6(\mathrm{X}=\mathrm{Cl}), 102.7(\mathrm{X}=\mathrm{Br})$ 和 $100.9 \mathrm{~kJ} \cdot \mathrm{mol}^{-1}(\mathrm{X}=\mathrm{I})$. 方法对比发现，只有这个方法和基组所得数据与 Bolm 等 ${ }^{[47]}$ 在实验中观察到反应活性大小顺序 $\mathrm{F}>\mathrm{I}>\mathrm{Br}>\mathrm{Cl}$ 最为接近. 由于 $102.7(\mathrm{X}=\mathrm{Br})$ 和 $100.9 \mathrm{~kJ} \cdot \mathrm{mol}^{-1}(\mathrm{X}=\mathrm{I})$ 数据比较接近, 怀疑可能是计算水平误差, 因此我们在 $\mathrm{MP} 3 / 6-311+\mathrm{G}^{* *} / / \mathrm{B} 3 \mathrm{LYP} / 6-311+\mathrm{G}^{* *}$ 水平上做了较高 级别的液相单点能计算, 结果发现三重微扰方法并没有 拉开二者之间的距离，因此以下的讨论均采用 MP2/6-311 $+\mathrm{G}^{* *} / / \mathrm{B} 3 \mathrm{LYP} / 6-311+\mathrm{G}^{* *}$ 方法水平上所得 计算结果.

表 $1 \mathrm{DMSO}$ 中标题反应所有反应物 $\mathbf{R e}$ 与其自由基阴离子相对能量表 $\left(\mathrm{kJ}^{\mathrm{N}} \cdot \mathrm{mol}^{-1}\right)$

Table 1 Relative energies of all the radical anions relative to reactants $\mathbf{R e}$ in DMSO of title reactions $\left(\mathrm{kJ}^{\circ} \mathrm{mol}^{-1}\right)$

\begin{tabular}{|c|c|c|c|c|}
\hline \multirow{2}{*}{ Single free radical } & \multicolumn{4}{|c|}{$\Delta G^{a} /\left(\mathrm{kJ} \cdot \mathrm{mol}^{-1}\right)$} \\
\hline & $\mathrm{F}$ & $\mathrm{Cl}$ & $\mathrm{Br}$ & $\mathrm{I}$ \\
\hline $\mathrm{M} 06-2 \mathrm{X} / 6-311+\mathrm{G}^{* *}$ & 466.6 & 349.9 & 308.3 & 263.8 \\
\hline 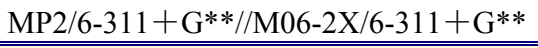 & 557.8 & 454.9 & 394.8 & 351.5 \\
\hline \multirow{2}{*}{ Diradical (triplet state) } & \multicolumn{4}{|c|}{$\Delta G^{b} /\left(\mathrm{kJ} \cdot \mathrm{mol}^{-1}\right)$} \\
\hline & $\mathrm{F}$ & $\mathrm{Cl}$ & $\mathrm{Br}$ & $\mathrm{I}$ \\
\hline $\mathrm{M} 06-2 \mathrm{X} / 6-311+\mathrm{G}^{* *}$ & 275.5 & 260.0 & 260.4 & 261.7 \\
\hline $\mathrm{MP} 2 / 6-311+\mathrm{G}^{* *} / / \mathrm{M} 06-2 \mathrm{X} / 6-311+\mathrm{G}^{* *}$ & 463.3 & 443.1 & 435.5 & 431.8 \\
\hline
\end{tabular}

${ }^{a} \Delta G=G(\mathbf{1 - 1})+G(\mathbf{X} \cdot)-G(\mathbf{R e}) ;{ }^{b} \Delta G=G(\mathbf{2}-\mathbf{1})-G(\mathbf{R e})$.

表 2 不同水平上计算所得标题反应 $\mathrm{S}_{\mathrm{N}} \mathrm{Ar}$ 路径反应能垒表

Table 2 Reaction energy barriers for the title reactions in the $S_{N} A r$ pathway calculated at different level

\begin{tabular}{|c|c|c|c|c|c|c|c|c|c|c|c|c|}
\hline \multirow{2}{*}{ Level } & \multicolumn{4}{|c|}{$\Delta E^{\ddagger} /\left(\mathrm{kJ} \bullet \mathrm{mol}^{-1}\right)$} & \multicolumn{4}{|c|}{$\Delta H^{\ddagger} /\left(\mathrm{kJ} \cdot \mathrm{mol}^{-1}\right)$} & \multicolumn{4}{|c|}{$\Delta G^{\ddagger} /\left(\mathrm{kJ} \cdot \mathrm{mol}^{-1}\right)$} \\
\hline & $\mathrm{F}$ & $\mathrm{Cl}$ & $\mathrm{Br}$ & I & $\mathrm{F}$ & $\mathrm{Cl}$ & $\mathrm{Br}$ & I & $\mathrm{F}$ & $\mathrm{Cl}$ & $\mathrm{Br}$ & I \\
\hline B3LYP/6-31+G* & 121.6 & 128.4 & 119.3 & 121.4 & 115.8 & 123.5 & 114.9 & 117.0 & 120.8 & 127.9 & 120.3 & 120.2 \\
\hline B3LYP/6-311+G** & 118.7 & 128.9 & 122.6 & 120.6 & 113.1 & 124.1 & 118.5 & 116.4 & 119.6 & 132.5 & 122.9 & 120.2 \\
\hline $\mathrm{M} 062 \mathrm{X} / 6-311+\mathrm{G}^{* *}$ & 113.2 & 126.0 & 125.2 & 112.1 & 109.1 & 124.1 & 120.7 & 106.2 & 115.5 & 128.5 & 127.2 & 116.1 \\
\hline $\begin{array}{l}\mathrm{MP} 2 / 6-311+\mathrm{G}^{* *} / / \\
\mathrm{M} 062 \mathrm{X} / 6-311+\mathrm{G}^{* *}\end{array}$ & 94.2 & 102.5 & 96.3 & 82.6 & 87.9 & 97.2 & 91.8 & 78.7 & 94.3 & 101.6 & 98.4 & 88.6 \\
\hline $\begin{array}{l}\text { MP2/6-311+G**// } \\
\text { B3LYP/6-311+ } \text { G** }^{* *}\end{array}$ & 89.6 & 106.0 & 102.4 & 101.4 & 84.0 & 101.2 & 98.3 & 97.2 & 90.5 & 109.6 & 102.7 & 100.9 \\
\hline $\begin{array}{l}\mathrm{MP} 3 / 6-311+\mathrm{G}^{* *} / / \\
\mathrm{B} 3 \mathrm{LYP} / 6-311+\mathrm{G}^{* *}\end{array}$ & 126.8 & 146.6 & 141.1 & 140.3 & 121.4 & 141.6 & 136.9 & 136.1 & 127.9 & 150.0 & 141.3 & 139.9 \\
\hline
\end{tabular}




\section{3 标题反应的反应性顺序解释}

通常饱和卤代烃协同 $\mathrm{S}_{\mathrm{N}} 2$ 亲核取代反应的能垒与 $\mathrm{C}-\mathrm{X}$ 键极性大小顺序 $\mathrm{X}=\mathrm{F}>\mathrm{Cl}>\mathrm{Br}>\mathrm{I}$ 一致 ${ }^{[76]}$. 最近 我们研究得出, 没有活化的卤代芳烃上的 $\mathrm{S}_{\mathrm{N}} \mathrm{Ar}$ 亲核取 代反应的能垒同样与 $\mathrm{C}-\mathrm{X}$ 键极性大小顺序 $\mathrm{X}=\mathrm{F}>$ $\mathrm{Cl}>\mathrm{Br}>\mathrm{I}$ 一致 ${ }^{[77]}$. 而标题反应的反应活性顺序却是 $\mathrm{F}>\mathrm{I}>\mathrm{Br}>\mathrm{Cl}^{[51]}$, 究竟是什么因素控制着反应的能垒? 本部分将通过几何结构宽松度分析、NPA 电荷分析以及 反应性指标分析对标题反应的反应活性顺序作出解释.

\subsection{1 几何结构宽松度分析}

从图 4 可以看出, 标题反应协同 $\mathrm{S}_{\mathrm{N}} \mathrm{Ar}$ 路径(Path 4) 过渡态中主要的几何结构变化特征为 $\mathrm{X} 1-\mathrm{C} 2$ 和 $\mathrm{C} 2-$ N10 单键相对于反应物与产物复合物或产物的键长变 化. 我们可以用 Shaik 等 ${ }^{\left[{ }^{[7]}\right.}$ 提出的几何结构宽松度(键长 变化的百分数)的概念来描述过渡态中 $\mathrm{X} 1-\mathrm{C} 2$ 和 $\mathrm{C} 2-$ $\mathrm{N} 10$ 单键的变化情况, $\left(\mathrm{X} 1-\mathrm{C}^{\ddagger}\right) \%,\left(\mathrm{C} 2-\mathrm{N} 10^{\ddagger}\right) \%$ 与 $L \%$ 定义为:

$$
\begin{aligned}
& \left(\mathrm{X} 1-\mathrm{C} 2^{\ddagger}\right) \%=100\left[R^{\ddagger}(\mathrm{X} 1-\mathrm{C} 2)-R^{\mathrm{Re}}(\mathrm{X} 1-\mathrm{C} 2)\right] / \\
& R^{\mathrm{Re}}(\mathrm{X} 1-\mathrm{C} 2)
\end{aligned}
$$

$$
\begin{aligned}
& \left(\mathrm{C} 2-\mathrm{N} 10^{\ddagger}\right) \%=100\left[R^{\ddagger}(\mathrm{C} 2-\mathrm{N} 10)-R^{\mathrm{Pro}}(\mathrm{C} 2-\right. \\
& \mathrm{N} 10)] / R^{\mathrm{Pro}}(\mathrm{C} 2-\mathrm{N} 10) \\
& L \%=\left(\mathrm{X} 1-\mathrm{C} 2^{\ddagger}\right) \%+\left(\mathrm{C} 2-\mathrm{N} 10^{\ddagger}\right) \%
\end{aligned}
$$

这里 $R^{\neq}, R^{\mathrm{Re}}$ 和 $R^{\mathrm{Pro}}$ 分别为过渡态结构 $\mathrm{TS}$ 中的键长, 反 应物 $\operatorname{Re}$ 和产物复合物或产物 Pro 中的键长. 很明显, 假 如 $\left(\mathrm{X} 1-\mathrm{C}^{\ddagger}\right) \%,\left(\mathrm{C} 2-\mathrm{N} 10^{\neq}\right) \%$ 或 $L \%$ 值越大，则反应能垒 越大. 计算所得几何结构宽松度 $\left(\mathrm{X} 1-\mathrm{C}^{\mp}\right) \%,(\mathrm{C} 2-$ $\left.\mathrm{N} 10^{\neq}\right) \%$ 和 $L \%$ 值总结在表 3 中, 从表 3 数据可以看出, 不 管是 $\left(\mathrm{X} 1-\mathrm{C}^{\ddagger}\right) \%,\left(\mathrm{C} 2-\mathrm{N} 10^{\ddagger}\right) \%$ 还是 $L \%$ 值, 大小顺序为 $\mathrm{I}>\mathrm{Br}>\mathrm{Cl}>\mathrm{F}$, 因此反应性顺序理论上应该是 $\mathrm{F}>\mathrm{Cl}>$ $\mathrm{Br}>\mathrm{I}$, 然而标题反应的反应活性顺序却是 $\mathrm{F}>\mathrm{I}>\mathrm{Br}>$ $\mathrm{Cl}$. 故几何结构宽松度分析不能解释标题反应反应性大 小顺序问题.

\subsubsection{NPA 电荷分析}

对于亲核反应来说，如果亲核试剂所带负电荷越多 而被进攻原子所带正电荷越多，则反应的能垒就越低， 反应就越容易进行. 我们在 MP2/6-311+ $\mathrm{G}^{* * / / B 3 L Y P / ~}$ 6-311 $+\mathrm{G}^{* *}$ 水平上计算得到主要原子 NPA 电荷数据总 结在表 4 中.

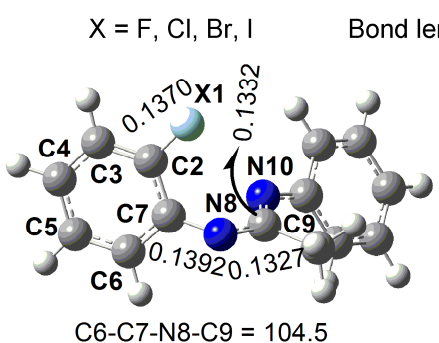

F-Re

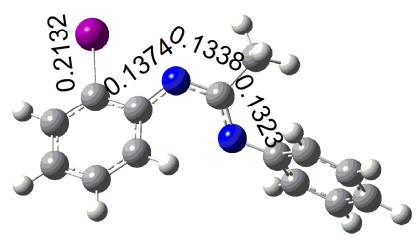

C6-C7-N8-C9 = 39.2

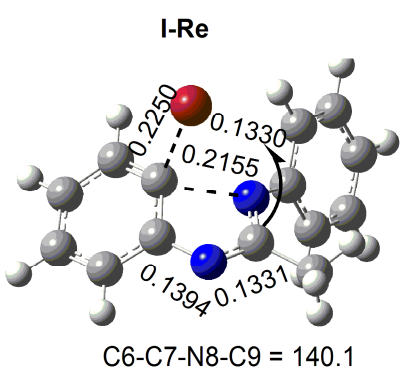

Br-TS

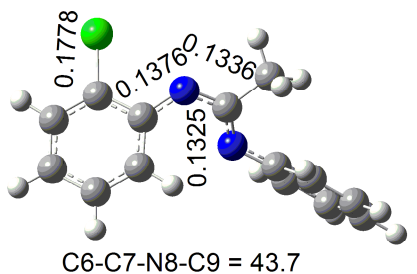

Cl-Re

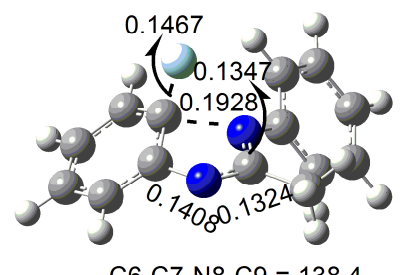

F-TS

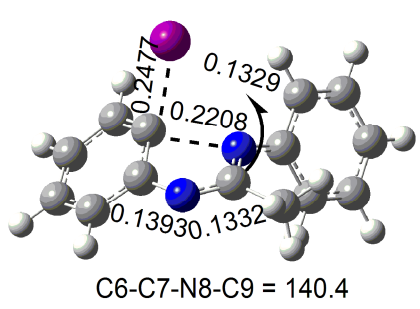

I-TS

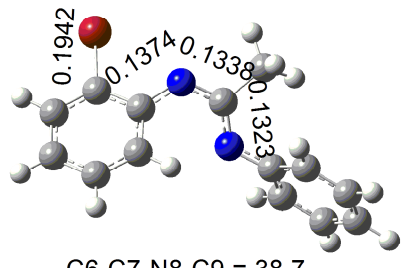

C6-C7-N8-C9 $=38.7$

Br-Re

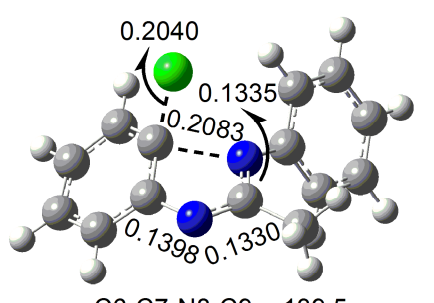

C6-C7-N8-C9 = 139.5

CI-TS

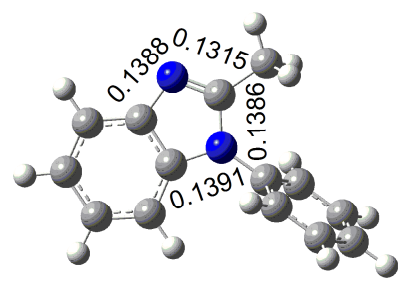

Pro

图 4 DMSO 中 B3LYP/6-311+ $\mathrm{G}^{* *}$ 水平上优化所得标题反应协同 $\mathrm{S}_{\mathrm{N}} \mathrm{Ar}$ 路径所有反应物、过渡态和产物几何结构

Figure 4 B3LYP/6-311 $+\mathrm{G}^{* *}$ optimized all the geometry structures of reactant, TS and product in DMSO of title reactions in the concerted $\mathrm{S}_{\mathrm{N}} \mathrm{Ar}$ pathway 
表 3 协同 $\mathrm{S}_{\mathrm{N}} \mathrm{Ar}$ 路径过渡态几何结构宽松 $\left(\mathrm{X} 1-\mathrm{C}^{\ddagger}\right) \%$ 和 $\quad\left(\mathrm{C} 2-\mathrm{N} 10^{\ddagger}\right) \%$ 以及 $\mathrm{L} \%$ 值与能垒

Table 3 TS geometric looseness of the $\mathrm{X} 1-\mathrm{C} 2$ and $\mathrm{C} 2-\mathrm{N} 10$ bonds by the parameters $\left(\mathrm{X} 1-\mathrm{C} 2^{\ddagger}\right) \%$, $\left(\mathrm{C} 2-\mathrm{N} 10^{\ddagger}\right) \%$ and $L \%$ and the TS energy barriers in $\mathrm{S}_{\mathrm{N}} \mathrm{Ar}$ pathway of title reactions

\begin{tabular}{cccccc}
\hline $\mathrm{X}$ & $\left(\mathrm{X} 1-\mathrm{C} 2^{\ddagger}\right) \%$ & $\left(\mathrm{C} 2-\mathrm{N} 10^{\ddagger}\right) \%$ & $L / \%$ & $\Delta E^{\ddagger} /\left(\mathrm{kJ} \cdot \mathrm{mol}^{-1}\right)$ & $\Delta G^{\ddagger} /\left(\mathrm{kJ} \cdot \mathrm{mol}^{-1}\right)$ \\
\hline $\mathrm{F}$ & 7.0 & 38.4 & 45.4 & 89.5 & 90.7 \\
$\mathrm{Cl}$ & 14.8 & 49.7 & 64.4 & 105.8 & 109.6 \\
$\mathrm{Br}$ & 15.9 & 54.9 & 70.8 & 102.5 & 102.9 \\
$\mathrm{I}$ & 16.2 & 58.7 & 74.9 & 101.2 & 100.8 \\
\hline
\end{tabular}

表 4 MP2 $/ 6-311+\mathrm{G}^{* * / / B} 3 \mathrm{LYP} / 6-311+\mathrm{G}^{* *}$ 水平上计算所得主要原子 $\mathrm{NPA}$ 电荷 $(Q)$

Table 4 Main atomic NPA charges $(Q)$ calculated at MP2/6-311+ $\mathrm{G}^{* *} / / \mathrm{B} 3 \mathrm{LYP} / 6-311+\mathrm{G}^{* *}$ level

\begin{tabular}{|c|c|c|c|c|c|c|c|c|c|c|c|c|}
\hline \multirow{2}{*}{$\mathrm{X}$} & \multicolumn{2}{|c|}{$\mathrm{X} 1$} & \multicolumn{2}{|c|}{$\mathrm{C} 2$} & \multicolumn{2}{|c|}{ C7 } & \multicolumn{2}{|c|}{ N8 } & \multicolumn{2}{|c|}{ C9 } & \multicolumn{2}{|c|}{ N10 } \\
\hline & $\mathbf{R e}$ & TS & $\operatorname{Re}$ & TS & $\operatorname{Re}$ & TS & $\mathbf{R e}$ & TS & $\mathbf{R e}$ & TS & $\mathbf{R e}$ & TS \\
\hline $\mathrm{F}$ & -0.4271 & -0.5100 & 0.4021 & 0.5735 & 0.1712 & 0.0255 & -0.8354 & -0.7532 & 0.6142 & 0.5970 & -0.8402 & -0.7281 \\
\hline $\mathrm{Cl}$ & -0.0777 & -0.3445 & -0.0878 & 0.2585 & 0.2243 & 0.0765 & -0.8318 & -0.7813 & 0.6186 & 0.6147 & -0.8164 & -0.7485 \\
\hline $\mathrm{Br}$ & 0.0098 & -0.3443 & -0.1897 & 0.2228 & 0.2266 & 0.0892 & -0.8321 & -0.7886 & 0.6196 & 0.6186 & -0.8118 & -0.7577 \\
\hline I & 0.1186 & -0.3075 & -0.2898 & 0.1807 & 0.2258 & 0.0880 & -0.8355 & -0.7885 & 0.6200 & 0.6200 & -0.8106 & -0.7682 \\
\hline
\end{tabular}

从表 4 可以看出, 在反应物 $\mathbf{R e}$ 中, 亲核原子 $\mathrm{N} 10$ 所带负电荷依次减少, 分别为 $-0.8402 e(\mathrm{X}=\mathrm{F}),-$ $0.8164 e(\mathrm{X}=\mathrm{Cl}),-0.8118 e(\mathrm{X}=\mathrm{Br}),-0.8106 e(\mathrm{X}=\mathrm{I})$; 而被进攻原子 $\mathrm{C} 2$ 所带正电荷也是依次减少, 分别为 $0.4021 e(\mathrm{X}=\mathrm{F}),-0.0878 e(\mathrm{X}=\mathrm{Cl}),-0.1897 e(\mathrm{X}=\mathrm{Br})$ 和 $-0.2898 e(\mathrm{X}=\mathrm{I})$, 因此标题反应的反应性顺序理论 上应该是 $\mathrm{F}>\mathrm{Cl}>\mathrm{Br}>\mathrm{I}$, 结果与几何结构宽松度分析一 致, 同样与 Bolm 等实验结论 ${ }^{[51]}$ 不符. 因此 NPA 电荷分 析也不能解释标题反应反应性大小顺序问题.

\subsection{3 反应性指标分析}

从图 5 可以看出, 标题反应电子能垒 $\Delta E^{\ddagger}\left(\mathrm{kJ} \cdot \mathrm{mol}^{-1}\right)$ 和吉布斯自由能垒 $\Delta G^{\neq}\left(\mathrm{kJ} \bullet \mathrm{mol}^{-1}\right)$ 之间呈现很好的线性 关系 $\left(R^{2}=0.97486\right)$, 因此我们可以用 $\Delta E^{\ddagger}$ 来代替 $\Delta G^{\ddagger}$ 来 分析标题反应反应性大小顺序问题.

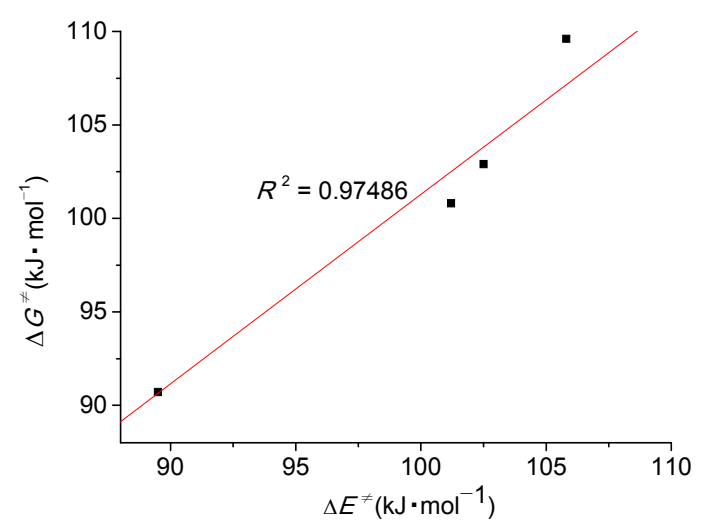

图 5 标题反应电子能垒 $\left(\Delta E^{\ddagger}\right)$ 和吉布斯自由能垒 $\left(\Delta G^{\ddagger}\right)$ 关系图 Figure 5 Plot of electronic barriers $\left(\Delta E^{\ddagger}\right)$ vs the Gibbs free energy barriers $\left(\Delta G^{\ddagger}\right)$ of the title reactions

进一步研究发现, 运用反应性指标理论 ${ }^{[80 \sim 85]}$ 或许 可以很好地解释标题反应的反应性大小顺序问题. 该理
论曾被其他课题组成功地用来解释芳环上的亲核取代 反应顺序 ${ }^{[76,77]}$. 根据反应性指标理论，亲核试剂的亲核 性指标可以近似用下式表示 ${ }^{[75 ~ 77]}: \omega^{-}=-I \approx E_{\mathrm{HOMO}}$ ，定 域亲核性指标表示为 ${ }^{[80]}: \omega^{-}(k)=\omega^{-} f^{-}(k)$. 其中 $\omega^{-}$和 $I$ 以 及 $E_{\mathrm{HOMO}}$ 分别为亲核试剂亲核性指标、离子势和最高占 据轨道能; $\omega^{-}(k)$ 和 $f^{-}(k)$ 表示 $k$ 原子的定域亲核性指标和 $k$ 原子的定域亲核福井函数. 而定域亲核福井函数表示 为 ${ }^{[83 \sim 85]}: f^{-}(k)=P_{k}(N)-P_{k}(N-1) . P_{k}(N)$ 和 $P_{k}(N-1)$ 表示 含 $N$ 个电子系统中 $k$ 原子的 NPA 电荷以及当此系统失 掉一个电子后系统中 $k$ 原子的 NPA 电荷.

根据反应性指标理论, $\omega^{-}$值越小或 $\omega^{-}(k)$ 值越大, 反应物的亲核性越强, 反应能垒越低, 反应性越强. 我 们计算得出, 各反应物最高占据轨道能 $\left(E_{\mathrm{HOMO}}, \mathrm{eV}\right)$ 和 $\omega^{-} \mathrm{N} 10(\mathrm{eV})$ 分别与反应电子能垒 $\Delta E^{\ddagger}\left(\mathrm{kJ} \cdot \mathrm{mol}^{-1}\right)$ 和吉布斯 自由能垒 $\Delta G^{\ddagger}\left(\mathrm{kJ} \bullet \mathrm{mol}^{-1}\right)$ 呈现较好的线性关系 $\left(R^{2}=\right.$ $0.96811,0.97102$ 和 0.95716 , 图 6,7), 因此可以得出标 题反应的反应性主要是由最高占据轨道能 $\left(E_{\mathrm{HOMO}}\right)$ 控制.

从以上分析可知, 标题反应中亲核试剂的亲核能力 可以由最高占据轨道能 $E_{\mathrm{HOMO}}$ 或者区域亲核性指标 $\omega^{-} \mathrm{N} 10$ 来分析, 理论上反应性大小还与 $\mathrm{C} 2$ 和 $\mathrm{N} 10$ 原子所 带电荷以及几何结构宽松度 $L \%$ 值有关.

$$
\begin{aligned}
& \Delta E^{\neq}= 1256.67 E_{\mathrm{HOMO}}-78.72 Q(\mathrm{C} 2)-1.59 L \%+ \\
& 354.7, R^{2}=1 \\
& \Delta E^{+}= 59391.69 E_{\mathrm{HOMO}}-50013.2 Q(\mathrm{~N} 10)+43.9 L \%- \\
& 36298, R^{2}=1 \\
& \Delta E^{+}=-1756.56 \omega^{-}{ }_{\mathrm{N} 10}-72.69 Q(\mathrm{C} 2)-1.83 L \%+ \\
& 297.78, R^{2}=1 \\
& \Delta E^{+}=-18266.2 \omega^{-}{ }_{\mathrm{N} 10}-10161.1 Q(\mathrm{~N} 10)+5.16 L \%- \\
& \\
& 7683.8, R^{2}=1
\end{aligned}
$$



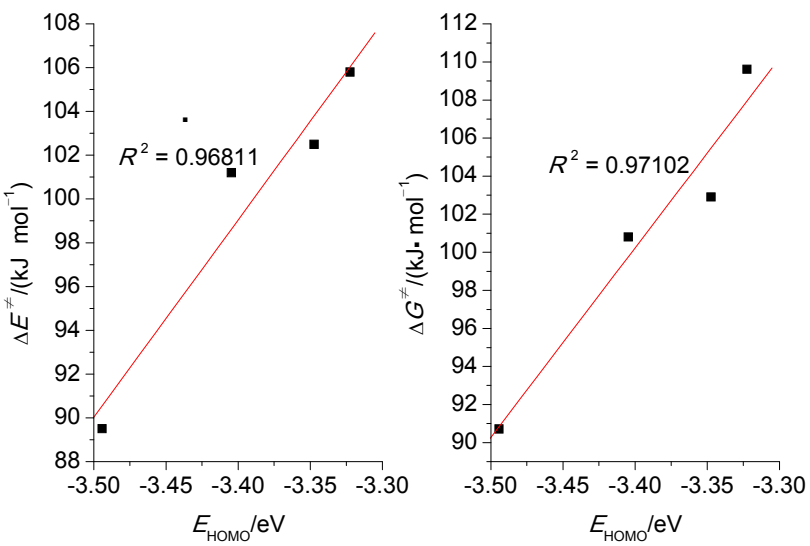

图 6 各反应物最高占据轨道能 $\left(E_{\mathrm{HOMO}}\right)$ 与反应电子能垒 $\Delta E^{\ddagger}$ 和吉布斯自由能垒 $\Delta G^{\ddagger}$ 关系图

Figure 6 Plot of the highest occupied molecular orbital energy $\left(E_{\mathrm{HOMO}}\right)$ of the reactants vs electronic barriers $\left(\Delta E^{\ddagger}\right)$ and Gibbs free energy barriers $\left(\Delta G^{\ddagger}\right)$ of the title reactions

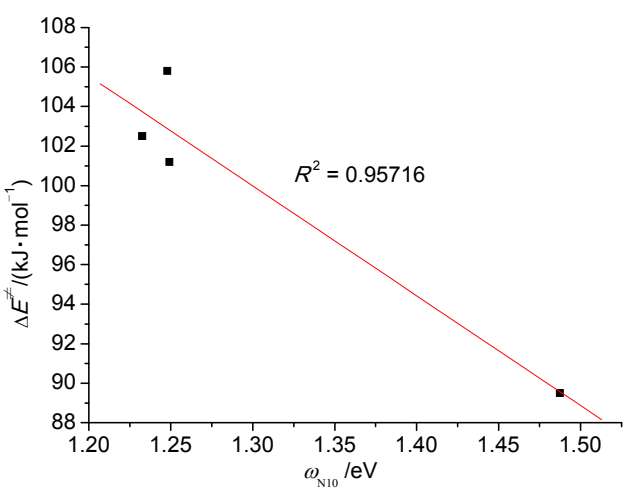

图 $7 \mathrm{~N} 10$ 原子的区域亲核性指标 $\left(\omega^{-} \mathrm{N} 10\right)$ 与反应电子能垒 $\Delta E^{\ddagger}$ 关系图

Figure 7 Plot of the regional nucleophilicity index of N10 atom $\left(\omega^{-} \mathrm{N} 10\right)$ vs electronic barriers $\left(\Delta E^{\ddagger}\right)$

表 5 最高占据轨道能 $\left(E_{\mathrm{HOMO}}, \mathrm{eV}\right)$ 、亲核原子 $\mathrm{N} 10$ 定域亲核 性指标 $\left(\omega^{-} \mathrm{N} 10, \mathrm{eV}\right)$ 和定域亲核福井函数 $f^{-} \mathrm{N} 10$ 以及反应能垒

Table 5 Highest occupied molecular orbital energy $\left(E_{\text {Hомо, }}\right.$ $\mathrm{eV})$, the regional nucleophilicity index $\left(\omega^{-} \mathrm{N} 10, \mathrm{eV}\right)$ and regional nucleophilic Fukui function $f_{\mathrm{N} 10}$ of the nucleophile $\mathrm{N} 10$ atom as well as the TS energy barriers in $\mathrm{S}_{\mathrm{N}}$ Ar pathway of title reactions

\begin{tabular}{cccccc}
\hline $\mathrm{X}$ & $E_{\text {Номо }}$ & $\omega^{-}{ }_{\mathrm{N} 10}$ & $f_{\mathrm{N} 10}$ & $\begin{array}{c}\Delta E^{\ddagger} / \\
\left(\mathrm{kJ} \bullet \mathrm{mol}^{-1}\right)\end{array}$ & $\begin{array}{c}\Delta G^{\ddagger} / \\
\left(\mathrm{kJ} \bullet \mathrm{mol}^{-1}\right)\end{array}$ \\
\hline $\mathrm{F}$ & -3.4929 & 1.4867 & -0.4256 & 89.5 & 90.7 \\
$\mathrm{Cl}$ & -3.3212 & 1.2476 & -0.3756 & 105.8 & 109.6 \\
$\mathrm{Br}$ & -3.3460 & 1.2321 & -0.3682 & 102.5 & 102.9 \\
$\mathrm{I}$ & -3.4036 & 1.2487 & -0.3669 & 101.2 & 100.8 \\
\hline
\end{tabular}

通过多参数拟合得到了以上 4 个式子，其 $R$ 平方值 都为 1 . 从以上 4 个式子可以看出它们各自对反应能垒 的贡献权重. 很明显, 反应能垒主要由最高占据轨道能 $E_{\mathrm{HOMO}}$ 或亲核原子 $\mathrm{N} 10$ 的区域亲核性指标 $\omega^{-} \mathrm{N} 10$ 决定, 另一因素为亲核原子 $\mathrm{N} 10$ 电荷 $Q(\mathrm{~N} 10)$ 大小, 而 $\mathrm{C} 2$ 原子
电荷 $Q(\mathrm{C} 2)$ 以及几何结构宽松度 $L \%$ 对反应能垒影响很 小.

\section{4 结论}

采用 $\mathrm{MP} 2 / 6-311+\mathrm{G}^{* * / / B} 3 \mathrm{LYP} / 6-311+\mathrm{G}^{* *}$ 理论方 法, 对强碱催化 $N$-(2-卤基苯基)- $N$ - -苯基乙脒分子内氮 芳基化合成苯并咪唑反应机理和反应性做了理论研究. 为了评价方法、基组以及能量的可靠性，采用密度泛函 B3LYP 和 M06-2X 理论方法以及 $6-31+\mathrm{G}^{*}$ 和 6-311+ $\mathrm{G}^{* *}$ 基组对所有物质几何结构在 DMSO 中进行优化, 获 得了标题反应各个驻点几何结构参数, 在 MP2/6-311+

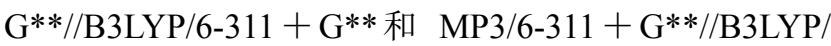

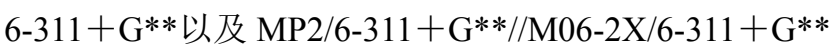
理论水平上进行了液相单点能计算, 最后得出结论如 下.

反应物 $\mathrm{Re}$ 中 $\mathrm{C}-\mathrm{X}$ 键发生均裂生成单自由基能垒 太高, 因此标题反应单自由基机理不可能存在; 另外, 只存在三重态双自由基，而单重态双自由基不存在，并 且在反应物 $\operatorname{Re}$ 中亲核原子 $\mathrm{N}$ 的 $\mathrm{p}$ 轨道与亲核基团苯环 的 $\pi$ 轨道存在 $\mathrm{p}-\pi$ 相互作用，而三重态双自由基阴离子 中却不存在这种作用, 导致后者能量比前者高出太多, 因此标题反应双自由基机理也不存在.

从过渡态结构以及反应能垒大小顺序可以得出，标 题反应的反应机理不是通常的分步 $\mathrm{S}_{\mathrm{N}} \mathrm{Ar}$ 机理, 而是只 有一个过渡态的协同 $\mathrm{S}_{\mathrm{N}} \mathrm{Ar}$ 机理.

相比于其他方法和基组, MP2/6-311+ $\mathrm{G}^{* * / / B 3 L Y P /}$ 6-311 $+\mathrm{G}^{* *}$ 方法能更好地描述标题反应的相对能量和 反应性顺序.

几何结构宽松度分析和 NPA 电荷分析都不能解释 标题反应的反应性大小顺序问题. 多参数拟合揭示标题 反应的反应能垒主要由最高占据轨道能 $E_{\mathrm{HOMO}}$ 或亲核原 子 $\mathrm{N} 10$ 的区域亲核性指标 $\omega^{-} \mathrm{N} 10$ 控制，另一因素为亲核 原子 $\mathrm{N} 10$ 所带负电荷的多少，而 $\mathrm{C} 2$ 原子电荷 $Q(\mathrm{C} 2)$ 以 及几何结构宽松度 $L \%$ 对反应能垒几乎没有影响.

辅助材料(Supporting Information) 文章中涉及的所 有结构的笛卡尔坐标和能量. 这些材料可以免费从本刊 网站(http://sioc-journal.cn/)上下载.

\section{References}

[1] Horton, D. A.; Bourne, G. T.; Smythe, M. L. Chem. Rev. 2003, 103, 893.

[2] Alamgir, M.; Black, D. St. C.; Kumar, N. Top. Heterocycl. Chem. 2007, 9, 87.

[3] Kedar, M. S.; Dighe, N. S.; Pattan, S. R.; Musmade, D. S.; Thakur D.; Bhosale, M.; Gaware, V. M. Pharma Chem. 2010, 2, 249.

[4] Srikanth, L.; Varun Raj, V.; Raghunandan, N.; Venkateshwerlu, L. 
Pharma Chem. 2011, 3, 172.

[5] Narasimhan, B.; Sharma, D.; Kumar, P. Med. Chem. Res. 2012, 21, 269.

[6] Lin, S. Y.; Isome, Y.; Stewart, E.; Liu, J. F.; Yohannes, D.; Yu, L. Tetrahedron Lett. 2006, 47, 2883.

[7] Dudd, L. M.; Venardou, E.; Garcia-Verdugo, E.; Licence, P.; Blake, A. J.; Wilson, C.; Poliakoff, M. Green Chem. 2003, 5, 187.

[8] Zhang, C.; Zhang, L.; Jiao, N. Green Chem. 2012, 14, 3273.

[9] Chari, M. A.; Shobha, D.; Sasaki, T. Tetrahedron Lett. 2011, 52, 5575.

[10] Riadi, Y.; Mamouni, R.; Azzalou, R.; Haddad, M. E.; Routier, S.; Guillaumet, G.; Lazar, S. Tetrahedron Lett. 2011, 52, 3492.

[11] Chari, M. A.; Shobha, D.; Kenawy, E. R.; Al-Deyab, S. S.; Reddy, B. V. S.; Vinu, A. Tetrahedron Lett. 2010, 51, 5195.

[12] Bahrami, K.; Khodaei, M. M.; Nejatia, A. Green Chem. 2010, 12, 1237.

[13] Wan, J. P.; Gan, S. F.; Wu, J. M.; Pan, Y. Green Chem. 2009, 11, 1633.

[14] Saha, D.; Saha, A.; Ranu, B. C. Green Chem. 2009, 11, 733.

[15] Bahrami, K.; Khodaei, M. M.; Naali, F. Synlett 2009, 569.

[16] Sharghi, H.; Aberi, M.; Doroodmand, M. M. Adv. Synth. Catal. 2008, 350, 2380.

[17] Mukhopadhyay, C.; Tapaswi, P. K. Tetrahedron Lett. 2008, 49, 6237.

[18] Bahrami, K.; Khodaei, M. M.; Naali, F. J. Org. Chem. 2008, 73, 6835.

[19] Zheng, N.; Anderson, K. W.; Huang, X.; Nguyen, H. N.; Buchwald, S. L. Angew. Chem., Int. Ed. 2007, 46, 7509.

[20] Zheng, N.; Buchwald, S. L. Org. Lett. 2007, 9, 4749.

[21] Zou, B.; Yuan, Q.; Ma, D. Angew. Chem., Int. Ed. 2007, 46, 2598.

[22] Diao, X.; Wang, Y.; Jiang, Y.; Ma, D. J. Org. Chem. 2009, 74, 7974.

[23] Kim, Y.; Kumar, M. R.; Park, N.; Heo, Y., Lee, S. J. Org. Chem. 2011, 76, 9577.

[24] Evindar, G.; Batey, R. A. Org. Lett. 2003, 5, 133.

[25] Brain, C. T.; Brunton, S. A. Tetrahedron Lett. 2002, 43, 1893.

[26] Brain, C. T.; Steer, J. T. J. Org. Chem. 2003, 68, 6814.

[27] Peng, J.; Ye, M.; Zong, C.; Hu, F.; Feng, L.; Wang, X.; Wang, Y.; Chen, C. J. Org. Chem. 2011, 76, 716.

[28] Brasche, G.; Buchwald, S. L. Angew. Chem., Int. Ed. 2008, 47, 1932.

[29] Xiao, Q.; Wang, W.; Liu, G.; Meng, F.; Chen, J.; Yang, Z.; Shi, Z. Chem. Eur. J. 2009, 15, 7292.

[30] Wray, B. C.; Stambuli, J. P. Org. Lett. 2010, 12, 4576.

[31] Deng, X.; Mani, N. S. Eur. J. Org. Chem. 2010, 4, 680.

[32] Shen, M.; Driver, T. G. Org. Lett. 2008, 10, 3367.

[33] Cheng, Z.; Zhang, Q. F.; Xu, X. L.; Li, X. N. Chin. J. Org. Chem. 2015, 35(6), 1189 (in Chinese). (程正, 张群峰, 许孝良, 李小年, 有机化学, 2015, 35(6), 1189.)

[34] Zhao, D. D.; Yu, J. T.; Wang, P. C.; Lu, M. Chin. J. Org. Chem. 2016, 36(1), 165 (in Chinese). (赵丹丹, 虞家涛, 王鹏程，陆明，有机化学，2016，36(1)，165.)

[35] Yu, Z. T.; Wang, Z. Y.; Wu, X.; Hu, G. Y.; Li, Q. B. Chin. J. Org. Chem. 2016, 36(7), 1672 (in Chinese). (余祖滔, 王泽瑜, 吴肖, 胡高云, 李乾斌, 有机化学, 2016, 36(7), 1672.)

[36] Meng, Y. X.; Gui, Y. Y.; Ji, Q.; Pan, Y.; L.; Lin, Z. Q.; Lü, L.; Zeng, X. C. Chin. J. Org. Chem. 2016, 36(2), 384 (in Chinese). (蒙玉霞, 桂显荣, 吉琼, 潘咏玲, 林志强, 吕柳, 曾向潮, 有机 化学, 2016, 36(2), 384.)

[37] Yuan, Y.; Thomé, I.; Kim, S. H.; Chen, D.; Beyer, A.; Bonnamour, J.; Zuidema, E.; Chang, S.; Bolm, C. Adv. Synth. Catal. 2010, 352, 2892 .
[38] Cano, R.; Ramón, D. J.; Yus, M. J. Org. Chem. 2011, 76, 654.

[39] Fang, Y.; Zheng, Y.; Wang, Z. Eur. J. Org. Chem. 2012, 7, 1495.

[40] Zou, L. H.; Reball, J.; Mottweiler, J.; Bolm, C. Chem. Commun. 2012, 48, 11307.

[41] Diness, F.; Fairlie, D. P. Angew. Chem., Int. Ed. 2012, 51, 8012.

[42] Carmen Pérez-Aguilar, M.; Valdés, C. Angew. Chem., Int. Ed. 2012, $51,5953$.

[43] Jalalian, N.; Petersen, T. B.; Olofsson, B. Chem.-Eur. J. 2012, 18, 14140.

[44] Majumdar, K. C.; Ganai, S.; Nandi, R. K.; Ray, K. Tetrahedron Lett. 2012, 53, 1553.

[45] Zhao, J.; Zhao, Y.; Fu, H. Angew. Chem., Int. Ed. 2011, 50, 3769.

[46] Beyer, A.; Reucher, C. M. M.; Bolm, C. Org. Lett. 2011, 13, 2876.

[47] Thomé, I.; Bolm, C. Org. Lett. 2012, 14, 1892.

[48] Beyer, A.; Buendia, J.; Bolm, C. Org. Lett. 2012, 14, 3948.

[49] Thom, I.; Besson, C.; Kleine, T.; Bolm, C. Angew. Chem., Int. Ed. 2013, 52, 7509.

[50] Xiang, S. K.; Tan, W.; Zhang, D. X.; Tian, X. L.; Feng, C.; Wang, B. Q.; Zhao, K. Q.; Hu, P.; Yang, H. Org. Biomol. Chem. 2013, 11, 7271.

[51] Baars, H.; Beyer, A.; Kohlhepp, S. V.; Bolm, C. Org. Lett. 2014, 16, 536.

[52] Bunnett, J. F.; Zahler, R. E. Chem. Rev. 1951, 49, 273.

[53] Terrier, F. The $S_{N} A r$ Reactions: Mechanistic Aspects, in Modern Nucleophilic Aromatic Substitution, Wiley-VCH Verlag GmbH \& Co. KGaA, Weinheim, Germany 2013, pp. $1 \sim 84$.

[54] Hunter, A.; Renfrew, M.; Taylor, J. A.; Whitmore, J. M. J.; Williams, A. J. Chem. Soc., Perkin Trans. 2 1993, 1703.

[55] Fernandez, I.; Frenking, G.; Uggerud, E. J. Org. Chem. 2010, 75(9), 2971.

[56] Glukhovtsev, M. N.; Bach, R. D.; Laiter, S. J. Org. Chem. 1997, 62(12), 4036.

[57] Simkin, B. Y.; Gluz, E. B.; Glukhovtsev, M. N.; Minkin, V. I. J. Mol. Struct. (THEOCHEM) 1993, 284(1 2), 123.

[58] Becke, A. D. J. Chem. Phys. 1993, 98, 5648.

[59] Lee, C.; Yang, W.; Parr, R. G. Phys. Rev. B 1988, 37, 785.

[60] Miehlich, B.; Savin, A.; Stoll, H.; Preuss, H. Chem. Phys. Lett. 1989, 157, 200.

[61] Jr, J. R. P.; Veloso, D. P. Phys. Chem. Chem. Phys. 2008, 10, 1118.

[62] Cid, M. V. F.; Buijs, W.; Witkamp, G. J. Ind. Eng. Chem. Res. 2007, $46,941$.

[63] Gorelsky, S. I.; Lapointe, D.; Fagnou, K. J. Am. Chem. Sos. 2008, 130,10848 .

[64] Imoto, M.; Matsui, Y.; Takeda, M.; Tamaki, A.; Taniguchi, H.; Mizuno, K.; Ikeda, H. J. Org. Chem. 2011, 76, 6356.

[65] Toledo, R. O.; Santos, J. G.; Ríos, P.; Castro, E. A.; Campodónico, P. R.; Contreras, R. J. Phys. Chem. B 2013, 117, 5908.

[66] Toledo, R. O.; Contreras, R.; Tapiab, R. A.; Campodónico, P. R. Org. Biomol. Chem. 2013, 11, 2302.

[67] Du, L. J.; Wu, C. H.; Gu, H. H.; Li, J. J. Org. Chem. 2015, 35(8), 1726 (in Chinese). (杜丽娟, 吴彩虹, 顾红红, 李娟, 有机化学, 2015, 35(8), 1726.)

[68] Glukhovtsev, M. N.; Bach, R. D.; Laiter, S. J. Org. Chem. 1997, 62, 4036.

[69] Wadt, W. R.; Hay, P. J. J. Chem. Phys. 1985, 82, 284.

[70] Tomasi, J.; Persico, M. Chem. Rev. 1994, 94, 2027.

[71] Reed, A. E.; Curtiss, L. A.; Weinhold, F. Chem. Rev. 1988, 88, 899.

[72] Zhao, Y.; Truhlar, D. G. Theor. Chem. Acc. 2008, 120, 215.

[73] Sadowsky, D.; McNeill, K.; Cramer, C. J. Environ. Sci. Technol. 2014, 48, 10904.

[74] Cairns, A. G.; Senn, H. M.; Murphy, M. P.; Hartley, R. C. Chem. Eur. J. 2014, 20, 3742. 
[75] Frisch, M. J.; Trucks, G. W.; Schlegel, H. B.; Scuseria, G. E.; Robb, M. A.; Cheeseman, J. R.; Scalmani, G.; Barone, V.; Mennucci, B.; Petersson, G. A.; Nakatsuji, H.; Caricato, M.; Li, X.; Hratchian, H. P.; Izmaylov, A. F.; Bloino, J.; Zheng, G.; Sonnenberg, J. L.; Hada, M.; Ehara, M.; Toyota, K.; Fukuda, R.; Hasegawa, J.; Ishida, M.; Nakajima, T.; Honda, Y.; Kitao, O.; Nakai, H.; Vreven, T.; Montgomery, J. J. A; Peralta, J. E.; Ogliaro, F.; Bearpark, M.; Heyd, J. J.; Brothers, E.; Kudin, K. N.; Staroverov, V. N.; Kobayashi, R.; Normand. J.; Raghavachari, K.; Rendell, A.; Burant, J. C.; Iyengar, S. S.; Tomasi, J.; Cossi, M.; Rega, N.; Millam, J. M.; Klene, M.; Knox, J. E.; Cross, J. B.; Bakken, V.; Adamo, C.; Jaramillo, J.; Gomperts, R.; Stratmann, R. E.; Yazyev, O.; Austin, A. J.; Cammi, R.; Pomelli, C.; Ochterski, J. W.; Martin, R. L.; Morokuma, K.; Zakrzewski, V. G.; Voth, G. A.; Salvador, P.; Dannenberg, J. J.; Dapprich, S.; Daniels, A. D.; Farkas, Ö.; Foresman, J. B.; Ortiz, J. V.; Cioslowski, J.; Fox, D. J. Gaussian 09, Revision A.02, Gaussian, Inc., Wallingford CT, 2009.

[76] Glukhovtsev, M. N.; Pross, A.; Radom, L. J. Am. Chem. Soc. 1996, $118,6273$.

[77] Li, Q. G.; Mao, S.; Cai, W. F.; Zheng, Y.; Liu, L. X. Chemistry
2016, 79(5), 418 (in Chinese).

(李强根, 毛双, 蔡皖飞, 郑妍, 刘柳斜, 化学通报, 2016, 79(5), 418.)

[78] Shaik, S. S.; Schlegel, H. B.; Wolfe, S. Theoretical Aspects of Physical Organic Chemistry. The $S_{N} 2$ Mechanism, Wiley, New York, 1992, pp. 181 188 .

[79] Reed, A. E.; Weinstock, R. B.; Weinhold, F. J. Chem. Phys. 1985, 83,735 .

[80] Chattaraj, P. K.; Sarkar, U.; Roy, D. R. Chem. Rev. 2006, 106, 2065.

[81] Ayers, P. W.; Anderson, J. S. M.; Bartolotti, L. J. Int. J. Quantum Chem. 2005, 101, 520.

[82] Contreras, R.; Andres, J.; Safont, V. S.; Campodonico, P.; Santos, J. G. J. Phys. Chem. A 2003, 107(29), 5588.

[83] Ormazábal-Toledo, R.; Contreras, R. Adv. Chem. 2014, 2014, 1.

[84] Ormazábal-Toledo, R.; Contreras, R.; Campodónico, P. R. J. Org. Chem. 2013, 78, 1091.

[85] Ormazábal-Toledo, R.; Campodónico, P. R.; Contreras, R. Org. Lett. 2011, 13, 822.

(Li, L.; Lu, Z.) 